\title{
TRABAJO Y SENSIBILIDADES: UN ANÁLISIS DE LA GESTIÓN DE LOS CUERPOS Y LAS EMOCIONES EN ALGUNOS ESPACIOS DE TRABAJO
}

\author{
Work and sensibilities: An analysis of management bodies and emotions in \\ workspaces
}

\author{
Pedro Lisdero' \\ Diego Quattrini
}

\section{Resumen}

El objetivo del trabajo es problematizar las consecuencias de la expansión del sistema capitalista en los procesos de sociabilidad del mundo del trabajo en el plano regional, desde una perspectiva que recupere los aportes de la teoría social sobre el cuerpo y las emociones. Se busca reflexionar sobre la "metamorfosis del mundo del trabajo", enfatizando el despliegue de las políticas de las sensibilidades que operan renovando condiciones para la re-configuración global de la mercantilización de los cuerpos y las emociones, en el siglo XXI. Para llevar a cabo el propósito se presentará un análisis de carácter cualitativo, analizando la experiencia de trabajadores de Call Centers y Emprendedores, en tanto experiencias paradigmáticas que dibujan algunas de las tendencias relevantes en la metamorfosis del paisaje cotidiano del trabajo, en las ciudades del Sur Global. El desarrollo argumentativo se abordará en dos momentos: en primer lugar, se realizará una breve revisión de la literatura sobre las transformaciones aludidas, a partir de la cual se definirán categorías teóricas relevantes para su análisis. Y en segundo lugar, se examinará las condiciones de la gestión del trabajo a partir de la exploración de entrevistas realizadas a trabajadores de Call Centers y "emprendedores", ambos de la provincia de Córdoba (Argentina).

Palabras-claves: Trabajo; Emoción; Sensibilidades.

\begin{abstract}
The objective of the paper is to problematize the consequences of the expansion of the capitalist system in the processes of sociability in the world of work at the regional level, from a perspective that recovers the contributions of social theory on the body and emotions. The aim is to reflect on the "metamorphosis of the world of work", emphasizing the deployment of policies of sensibilities that operate by renewing conditions for the global re-configuration of the commodification of bodies and emotions in the 21st century. To carry out the purpose, a qualitative analysis will be presented, analyzing the experience of Call Center workers and Entrepreneurs, as paradigmatic experiences that draw some of the relevant trends in the metamorphosis of the daily work landscape in the cities of the South Global. The argumentative development will be approached in two moments: first, a brief review of the literature on the aforementioned transformations will be carried out, from which relevant theoretical categories will be defined for its analysis. And secondly, the conditions of work management will be examined from the
\end{abstract}

\footnotetext{
1 Doctor en Estudios Sociales de América Latina, CEA-UNC. Lic. En Sociología, USXXI. Investigador Adjunto de CONICET - CIECS - UNC / Profesor Adjunto, UNVM.

2 Doctor en Ciencias Sociales, UNCuyo. Lic. En Sociología, USXXI. Profesor, UNVM. Profesor UPC.
} 
Trabajo y sensibilidades: Un análisis de la gestión de los cuerpos y las emociones en algunos espacios de trabajo | Pedro Lisdero \& Diego Quattrini

exploration of interviews carried out with Call Center workers and "entrepreneurs", both from the province of Córdoba (Argentina).

Keywords: Work; Emotion; Sensibilities.

\section{Introducción}

El capitalismo, desde sus inicios, ha procurado delimitar las corporalidades productivas y gestionar las emociones como componentes centrales de las lógicas disciplinares ${ }^{3}$. A lo largo del tiempo ha administrado un cúmulo de deseos y expectativas, que han promovido grados de aceptación y de articulación de formas típicas de sociabilidades necesarias para expandir una economía mercantil. Aun así, cada momento histórico ha tenido ciertas variaciones en los modos de consumir y poner al servicio de la producción las capacidades de los trabajadores -encarnadas tanto en emociones como en saberes cognitivos-. Para mantener y expandir su acumulación, el capitalismo occidental tuvo al cuerpo como el locus preferencial de dominación, procurando elaborar estrategias para definir y reproducir energias; exponiéndolo a la extracción más intensiva de un "plus-valor" que asegure saldos gananciales (SCRIBANO, 2016).

En este sentido, las diferentes estrategias de dominio del capital fueron (y están) vinculadas tanto a las políticas históricas específicas de disposición sobre los sistemas de pensamiento, acción y percepción como al intento de articulación entre estas y las formas de valorización del capital. Así, la concepción de capitalismo occidental permite enmarcar las instituciones históricas en la articulación con los arreglos específicos producidos en cada territorio en particular. La empresa, como expresión hegemónica del "lugar del

\footnotetext{
${ }^{3}$ Por su parte, la teoría social ha expresado tempranamente esta preocupación acerca del lugar de los cuerpos/emociones en la estructuración de la moderna sociedad capitalista. Así, es posible observar que los abordajes sociológicos sobre este temática se remontan a los "clásicos", Georg Simmel (BERICAT, 2001; Le Breton, 2002), Marx (Haber, 2007), pudiéndose incluir a autores como Erving Goffman (1989), Norbert Elias (1993), Pierre Bourdieu (1991) y Anthony Giddens (1991), entre otros. Como campo disciplinar, algunos autores ubican el origen de una sociología de los cuerpos en la obra de Brian Turner (GREMILION, 2005), en tanto que otros consideran a Thomas Scheff, Arlie Hochschild y Theodore Kemper como los pioneros en las investigaciones sociológicas sobre las emociones (BERICAT, 2000). En cuanto a un contexto más cercano, la aludida problemática sobre los cuerpos y las emociones cobra una creciente relevancia, abordando temáticas como las sexualidades (FIGARI, 2009; PECHENY, FIGARI y JONES, 2008; ACHA y HALPERIN, 2000), cuerpo-estética y arte (MATOSO, 2006; CITRO y ASCHIERI, 2012), sensibilidades (SCRIBANO y FIGARI, 2009; CERVIO, 2012; GALAK y DHERS, 2011; SCRIBANO y LISDERO, 2010; LUNA ZAMORA y SCRIBANO, 2007; SCRIBANO, 2007a, 2007b), entre otros.
} 
Trabajo y sensibilidades: Un análisis de la gestión de los cuerpos y las emociones en algunos espacios de trabajo | Pedro Lisdero \& Diego Quattrini

trabajo", junto con la existencia fáctica de una "sociedad salarial", son apenas algunos de los hitos de esta historia global que debe complejizarse al menos en dos direcciones específicas: la primera a tono con las particularidades en tiempo/espacio que implicó la yuxtaposición entre globalidad y neoliberalismo; y la segunda, con las metamorfosis del trabajo específicas territoriales de la periferia global, constituidas bajo una colonialidad del saber (QUIJANO, 2008) que produjo verdaderos obstáculos epistémicos para pensar los procesos históricos y los desbordes de las complejidades locales.

Se puede encontrar en las sociedades del Sur/Global un espacio social y una dimensión analítica relevante, para comprender las formas que adquiere la re-edición fundacional de la lógica de acumulación capitalista. Los cambios acontecidos en el "mundo del trabajo", entendidos de manera amplia como un conjunto de elementos que abarcan tanto las condiciones objetivas como el contenido específico de las subjetividades, proveen pistas analiticas para entender el quehacer de los sujetos de estos escenarios. Su configuración estuvo marcada tanto por una forma específica sobre la estructura de control del trabajo, sus recursos y sus productos (QUIJANO, 2008) como por la reinvención constante de las capacidades productivas.

En este contexto, los procesos de extracción global que promovieron consecuencias en lo local, tales como: la tercerización, la flexibilización en el pago de salarios, la informalización y racionalización del trabajo precario, el trabajo a destajo o la intervención de programas de gestión de los "recursos humanos y productivos no productivos", entre otros, no pueden ser explicados sin ponderar la incidencia de una serie de mecanismos y dispositivos orientados a la regulación de los cuerpos y las emociones de los sujetos-queviven-del-trabajo (ANTUNES, 2006). Adecuar estos cuerpos a las relaciones tecnológicas vinculadas a la gestión de las emociones, como al aparato productivo regional, constituye un proyecto político/económico/social que contribuye a la consolidación de una normatividad en la estructura geométrica y accional capitalista.

Desde este planteo, la dominación del sistema productivo remite a varias dimensiones, entre las que se encuentra la construcción de estructuras de poder y la elaboración de mecanismos dentro de los procesos que 
Trabajo y sensibilidades: Un análisis de la gestión de los cuerpos y las emociones en algunos espacios de trabajo | Pedro Lisdero \& Diego Quattrini

tradicionalmente definían los "ámbitos de trabajo", pero también fuera de estos. Esta es una de las claves relevantes para pensar la "metamorfosis global" del Sur Global, en donde los altos índices de informalidad, desocupación y marginalidad parecen consolidarse como condición de expansión de prácticas ligadas a las pretensiones del trabajo global. Estos espacios pueden ser analizado en función de la construcción de las sensibilidades en distintos ámbitos, las cuales re-inventan una sociabilidad enlazada a formas de consumo/producción, re-configurando no sólo los aspectos administrativos/organizacionales sino además la subjetividad y la entidad del propio cuerpo como sustrato privilegiado de estas transformaciones. Este proceso debe ser comprendido a partir del análisis del conjunto de las condiciones en que se despliega el metabolismo de las energías corporales laborales en capital (relaciones sociales), sin dejar de observar las consecuencias en la configuración de las políticas de las sensibilidades (SCRIBANO, 2009). Esta regulación emocional legitima la hegemonía en el mando en los procesos productivos, pero a su vez plantea la importancia del estudio de todas las relaciones y experiencias subjetivas y culturales que se construyen alrededor de los sentidos del trabajo. Esto supone una redefinición constante de aspectos cognitivos, morales, estéticos, sentimentales del sujeto en los comienzos del siglo XXI (DE LA GARZA, 2011). Las estrategias de quienes llevan adelante el espíritu de "técnico" empresarial -así como sus consecuencias- son un ejemplo de cómo las pedagogías de la administración del trabajo consideran como significativa la necesidad de elaborar una propuesta de organización, que conjugue la práctica laboral con el mundo de la vida, a fin de que los trabajadores adhieran, aunque sea mínimamente, a las reglas corporativas.

No resulta una casualidad la recurrencia a una sofisticación de formas con las que se genera la sujeción del trabajo, en la cual se instrumentalizan distintas doctrinas legitimas que procuran construir espacios culturales de acción y aprendizaje (FIGARI, 2011). Así la aparición y la renovación constante de "discursos morales empresariales" expresan un cuadro epocal, que muestra la forma en que se va configurando una fisonomía cognitiva y subjetiva de la modalidad de la explotación capitalista. Precisamente se 
Trabajo y sensibilidades: Un análisis de la gestión de los cuerpos y las emociones en algunos espacios de trabajo | Pedro Lisdero \& Diego Quattrini

observa una tensión en un número importante de sensaciones y emociones vitales de los trabajadores, las cuales son envueltas para la cooperación en el proceso de organización del trabajo (QUATTRINI, 2012). Esta carga emocional puede ser advertida también en las experiencias de millones de sujetos que transitan los espacios de informalidad, marginalidad social o precariedad laboral, donde se despliegan igualmente una serie de dispositivos y mecanismos que repercuten tanto en los labores y en las formas de percibirse como en sus capacidades. Estos compromisos emocionales, propios de los sujeto que transitan procesos de marginalidad, se articulan a partir de una serie de prácticas cotidianas consolidadas como mandatos de construcción identitaria asociados a los requerimientos del mercado, estructurando modos de ser y sentir que se reproducen en los contextos de exclusión (LISDERO; VERGARA, 2010). En este marco, sin menospreciar los aportes que desde diferentes campos referidos a los estudios sociales del trabajo, se analiza el lugar del cuerpo y las emociones en la configuración y gestión del trabajo actual. Para ello se propone un análisis de experiencias paradigmáticas con dos referencias empíricas del escenario del trabajo del Sur Global, (específicamente propio de América Latina). En este sentido se analizarán los procesos de trabajo, específicamente en su relación con la gestión del cuerpo y la emoción de los trabajadores de Call Centers y de aquellos denominados emprendedores que responden a un modelo de autoempleo/cuentapropista de sobrevivencia. La propuesta es observar en ambos grupos aquellas condiciones de trabajo, que construidas a partir de una territorialidad específica y bajo una organización del trabajo particular, proponen una expropiación de la capacidad operativa del ser social. Pero a su vez, se busca también identificar en estas experiencias la construcción del trabajo emocional, en tanto mecanismos donde se tensiona una creciente mercantilización de las sensibilidades, emociones y percepciones proyectadas en y para el trabajo. Las experiencias presentadas difieren entre sí, pero ambas revelan la consolidación de una definición productiva del cuerpo y las emociones, que despliega momentos de oclusión de conflictos y naturalización de formas de interacción. Esto da lugar a vivencias y creencias que reproducen el estado de sensibilidad social, que sirve como referencia a la normatividad 
Trabajo y sensibilidades: Un análisis de la gestión de los cuerpos y las emociones en algunos espacios de trabajo | Pedro Lisdero \& Diego Quattrini

de las exigencias que presenta el trabajo actual. En su conjunto, estas experiencias expresan una sola unidad analítica, donde cada una aporta de manera diferencial elementos significativos para caracterizar de manera paradigmáticas la sociabilidad transversal implicada en el vivir del trabajo, en el Sur Global, en el contexto del Siglo XXI.

El material empírico que se presentará en este artículo ha sido producido en el marco de dos líneas investigativas ${ }^{4}$, conducidas por los autores de este trabajo. Si bien cada una de las líneas comprendian objetivos y contextos de investigación específicos, ambas confluyen en ciertos "puntos de partida" comunes acerca: a. del modo de acumulación estructurado en los territorios del Sur Global, en el marco de la expansión de un capitalismo neocolonial; b. de la concepción de cuerpo (subjetivo, social e individual) tanto como del lugar que ocupa en los procesos de metamorfosis del trabajo; y cdel interés acerca de las emociones dentro de la estructuración de los mecanismos metabólicos del capital. Así, el fundamento general de la metodología, que aquí se propone, parte de reconocer la potencialidad del "análisis secundario de datos cualitativos", entendido como el procedimiento mediante el cual se utiliza información registrada en otros contextos, reconstruyendo su descripción y sistematización desde una estrategia de indagación complementaria a la original (SCRIBANO Y DE SENA, 2009, p. 105). Tal como observan Scribano y De Sena, las investigaciones con datos

\footnotetext{
${ }^{4}$ Una línea de investigación desarrollada por Pedro Lisdero, titulada "Acción colectiva, sensibilidades y trabajo. Conflictividades emergentes en el contexto de las nuevas morfologias del trabajo", e inscripta institucionalmente en el trabajo desarrollado como becario e investigador de CONICET, tuvo como principal objetivo caracterizar las conexiones entre acciones colectivas y sensibilidades sociales en trabajadores de experiencias emergentes vinculadas a la transformación del mundo del trabajo en la ciudad de Córdoba, Argentina. Así, desde 2006 a esta parte se ha conducido una estrategia de investigación que incluyó entrevistas en profundidad a trabajadores de Call Centers, registros obtenidos a partir de la observación de instancias públicas de protestas de estos actores y una extensa tarea de etnografia virtual (a partir de blogs, perfiles de Facebook, y otros soportes digitales utilizados por las organizaciones que protestan en el sector) (LISDERO, 2017). La segunda línea de investigación, denominada "Las dificultades del microemprendedor. Un análisis de Ventanilla del Emprendedor", dirigida por Diego Quattrini y financiada por la Universidad Nacional de Villa María para el periodo 2016-2017, tuvo como objetivo analizar las herramientas de gestión, las actitudes para el trabajo y el capital social de los emprendedores. A partir del 2015, se vienen desarrollando una serie de entrevistas en profundidad de tipo individual a fin de mapear las experiencias del emprender. Específicamente, estas entrevistas tienen la particularidad de realizarse en el lugar de trabajo y de utilizar la fotografia, como un medio complementario para favorecer la expresividad de los entrevistados (QUATTRINI, MARTÍN Y RAIMONDO, 2017).
} 
Trabajo y sensibilidades: Un análisis de la gestión de los cuerpos y las emociones en algunos espacios de trabajo | Pedro Lisdero \& Diego Quattrini

secundarios suelen ser subestimadas (a pesar de su relevancia, incluso para los clásicos de la sociología) o relegadas al campo de las investigaciones de tipo cuantitativas (2009, p. 102). Sin embargo, las investigaciones cualitativas más actuales comienzan a interesarse sobre las ventajas de la utilización de estas fuentes. En este caso, esta estrategia se ve facilitada por este "punto de partida" en común, el cual de alguna manera habilita la posibilidad de formular preguntas complementarias relacionadas a los objetivos de investigación, a partir de los cuales se construyeron las entrevistas.

En función de conocer más acerca de los contextos específicos de investigación aludidos, es posible aclarar que para las referencias a los trabajadores de Calls Centers, se elaboró un análisis de la expropiación corporal que se produce en estos espacios, y su vínculo con la regulación de las emociones (tomando al miedo como un nodo significativo). Se utilizó, como soporte material, una serie se extractos de entrevistas realizadas a trabajadores de la Ciudad de Córdoba en el periodo 2006-2017. Por otra parte, se tomó como referencia a los denominados emprendedores, a fin de examinar, a partir de sus experiencias en la precariedad, la regulación emocional que sustenta su trabajo (que incluye emociones como la tolerancia a la frustración y la tranquilidad, como la construcción de una impresión corporal). Aquí, se utilizaron, para el análisis, entrevistas a sujetos autodenominados emprendedores, en el periodo 2015-2018, en la Ciudad de Villa María (que forma parte de la provincia de Córdoba-Argentina) (QUATTRINI, MARTÍN Y RAIMONDO, 2017).

Así, teniendo en cuenta los contextos específicos en que se construyeron las entrevistas que se presentarán a continuación, se busca aquí identificar formas para conocer y analizar las emociones mediadas por las expresividades de los sujetos que trabajan. Se organizan "testimonios" que "expresen" emociones, sin pretensión comparativa ni representativa. El análisis, de estas experiencias emocionales de los emprendedores y de los trabajadores de Call Centers, brindó la oportunidad de observar la relación entre las condiciones estructurales capitalistas, las diversas mediaciones institucionales que poseen los distintos trabajos y las creencias particulares de los sujetos, a partir de vivencias paradigmáticas que enfatizan (de manera 
Trabajo y sensibilidades: Un análisis de la gestión de los cuerpos y las emociones en algunos espacios de trabajo | Pedro Lisdero \& Diego Quattrini

complementaria) algunas de las tendencias que aquí se pretenden poner en primer plano.

En función de esta propuesta general, la estrategia argumentativa que se seguirá a continuación responde a la siguiente organización. En primer lugar, se realizará una breve revisión de la literatura, a partir de la cual se definirán categorías teóricas relevantes para el análisis. En segundo lugar, se expondrán las experiencias de sujetos trabajadores en función de observar las condiciones de gestión del trabajo y el lugar que ocupan las emociones en esos procesos. Para finalizar, como apertura, se recuperará el planteo general a partir de pensar una "economía política de la moral" con características específicas, en contextos laborales del Sur Global propios del siglo XXI.

\section{I. "Metamorfosis" del "mundo del trabajo": aportes desde una sociología de los cuerpos/emociones}

Para acercarnos al análisis específico de los cuerpos/emociones, en el trabajo, es necesario realizar algunas referencias de los estudios sobre las transformaciones del mundo laboral. La amplia bibliografia, que remite a una sociología del trabajo, puede ser útil para caracterizar el régimen de acumulación (NUN, 2003) y la relación actual del capital y el trabajo, en base a las vicisitudes de los procesos económicos y sociales latinoamericanos generadas en los últimos años. Varios autores han reflexionado sobre las implicancias de los cambios en la constitución de la "clase que vive del trabajo" (ANTUNES, 2006) latinoamericana, observando: ciertas formas renovadas de la organización social del trabajo en actividades "modernas de la economía" (BRAGA, 2009; FIGARI, 2011); las consecuencias de los procesos de “externalización" y "mundialización" y la consolidación de la revolución tecnológica y su impacto (LISDERO, 2017); y la normalización del trabajo “precarizado", la cual remite a la institucionalización de la inestabilidad y la incertidumbre en el mundo actual (QUATTRINI, 2012).

Sintéticamente, estos procesos de reorganización de la producción pueden expresarse en la formación de un modelo de "acumulación flexible" (HARVEY, 2004), que posee como rasgo general la "perennidad" que presenta el trabajo y su oscilación hacia la superfluidad (ANTUNES, 2006). La perennidad se puede advertir en la heterogeneidad de la estructura 
Trabajo y sensibilidades: Un análisis de la gestión de los cuerpos y las emociones en algunos espacios de trabajo | Pedro Lisdero \& Diego Quattrini

productiva. Se observa así una creciente combinación de actividades que requieren la apropiación de una dimensión más cognitiva del trabajo y de habilidades sociales/afectivas que fomenten un ágil desenvolvimiento. Y junto a estas, en forma paralela, se visualiza además la ampliación del trabajo descalificado realizado por una fuerza de trabajo "sobrante" y superexplotada. Sin embargo, ambas poseen una demanda en ritmos e intensidad "emocional": tanto el trabajador formal de una moderna y "descontracturada" empresa productora de software, como el sujeto marginal que transita recogiendo cartón y botellas para vender como material reciclable, viven sus cotidianidades bajo la presión emocional de re-inventarse como "sujetos productivos".

La existencia de estos territorios heterogéneos altera las exigencias del mundo laboral y, específicamente, abre un espacio para comprender las conexiones entre estas "renovadas" condiciones de trabajo y el despliegue de un régimen de sensibilidades sociales. Millones de sujetos deben enfrentarse con los nuevos requerimientos propios de las innovaciones acontecidas. Emergen así mutaciones en los modos en que se valorizan las capacidades corporales (orgánicas, cognitivas, afectivas) de los trabajadores. En este sentido, el valor del trabajo, en muchas ramas económicas, no sólo está dado ahora en el acto objetivo de venta de la producción material, sino que necesita también del desarrollo de una dimensión "más" simbólica (DE LA GARZA, 2011).

La consolidación de la producción asociada a la mercantilización de las capacidades cognitivas y afectivas provoca entonces ciertos cambios en la clásica escisión de las dimensiones objetiva y subjetiva del trabajo. Aparece la problematización de la producción del sector servicio, trabajo que se objetiva principalmente en otro sujeto, cliente o usuario (y no en un objeto separado), colocando en el análisis la importancia del "trabajo emocional" (HOSCHCHILD, 1983).

Los nuevos debates, sobre los actuales procesos productivos, conllevan a la re-elaboración de significados de diversos órdenes y al análisis de las nuevas formas procedimentales: como son la elaboración de objetos de conocimientos con componentes simbólicos, la gestión de las emociones, la 
Trabajo y sensibilidades: Un análisis de la gestión de los cuerpos y las emociones en algunos espacios de trabajo | Pedro Lisdero \& Diego Quattrini

producción de valores morales o la valorización de aspectos estéticos ligados a lo corporal. Esto implica, a su vez, la consideración de la figura del "consumidor-cliente", demandando el desarrollo de nuevas capacidades, que incluyen la necesidad de agudizar sentidos orgánicos (vista, oído, gusto, olfato, tacto) a fin de lograr una mejor aproximación y de sentimientos adecuados para afrontar pedidos en "tiempos esperados" y con la "calidad" requerida según la presión solicitada (DURAND, 2011). En otras palabras, el principio del "cliente-rey", sumado a la exigencia creciente de la productividad obliga a acrecentar el esfuerzo, ahora expresado también en energías emocionales. Estos nuevos condicionamientos llevaron a reconocer la importancia que posee la gestión y el trabajo de control de las emociones, en la creación de valor de las nuevas morfologias de trabajo y sus consecuencias en el sistema afectivo, tal como lo señaló Hochschild (1983), a principios de la década de 1980.

Aquí la noción de "trabajo emocional", de Hoschchild, puede ser útil para observar la importancia de la capacidad de construcción de sensibilidades en su relación con los procesos de estructuración social. La autora aborda un análisis de las asistentes de vuelo y los vendedores de seguro (HOCHSCHILD, 1983), en donde conecta como eje de reflexión a las sensaciones producidas en el trabajo con los procesos de producción de valor. Expone el vínculo entre los requisitos laborales capitalistas contemporáneos, las exigencias en el sentir y el manejo de las emociones. Plantea que hay una institucionalización del orden emocional en el ámbito del trabajo que repercute en la esfera íntima/privada. Las emociones, al ser "gestionadas" en el mundo laboral, empiezan a poseer un cierto carácter "coactivo"; dejan de elaborarse a partir de un manejo discreto y personal; y pasa a ser un trabajo mercantilizarse a partir de una presentación adecuada en el ámbito público. $\mathrm{Su}$ estudio evidencia cómo muchos de los trabajadores no venden, simplemente, su fuerza física ni su conocimiento o capacidad racional, sino que colocan en el mercado también sus emociones.

Ante esta nueva situación, la autora propone como objeto de análisis un "cuerpo sensible", imbuido bajo una actuación estandarizada por normas y reglas laborales, cuerpo que posee al mismo tiempo la capacidad 
Trabajo y sensibilidades: Un análisis de la gestión de los cuerpos y las emociones en algunos espacios de trabajo | Pedro Lisdero \& Diego Quattrini

experimentar sensaciones, evaluar su adecuación y manejarlas de manera diestra en situaciones específicas (lo que le permite ocultar o provocar emociones). Ella utiliza el concepto de "actuación superficial" cuando el cuerpo moviliza expresiones como medio para modificar sus emociones; y "actuación profunda" cuando se "gestiona" la emoción, es decir, cuando se realiza el acto de tratar de cambiar en algún grado o cualidad lo que se siente (HOCHSCHILD, 1983) .

Quedan introducidas, en su análisis, las consecuencias de la incorporación de las emociones como propiedades de la fuerza de trabajo. Así, la mercantilización creciente de las emociones conduce a nuevas exigencias en el control y experimentación del cuerpo. Las relaciones en el trabajo demandan algo más que una puesta en escena, ya que en determinadas ocupaciones se exige disponer de un cuerpo con capacidades sensibles, que genere un saldo mercantilizado emocional. Esto exige una reconfiguración en la asunción de lo íntimo/privado, como una nueva consistencia en la acción gestual frente a los otros, lo que provoca una renovada forma de vivenciar el trabajo. Esta particularidad conduce al autoextrañamiento como vivencia cada vez más común de los sistemas capitalistas avanzados. Y si la repetición monótona de actos musculares operativos genera agotamiento, mucho más el trabajo emocional, ya que se debe aprender a reprimir e inducir estados de ánimos personales para generar amabilidad o soportar enfados de los clientes. De ahí que la racionalización de las energías emocionales crea condiciones de alienación y provoca cuerpos desgastados moralmente, destinados a la extracción de "personalidades" o a la formación de estados emocionales ficticios que entran en conflicto consigo mismo (BERICAT ALASTUEY, 2000).

Complejizando este último análisis, consideramos que este extrañamiento se constituye bajo la peculiaridad que posee la estructura capitalista neo-colonial latinoamericana (SCRIBANO, 2012), la cual va configurando las vivencialidades y las sensibilidades. Así, la estructura procedimental del capitalismo en su fase de "acumulación flexible" necesita de "regímenes de sensibilidades", que permitan la aceptación de la reproducción por parte de los trabajadores. Es decir, las exigencias continuas que genera el presente, de extenuación emocional del trabajo latinoamericano, 
Trabajo y sensibilidades: Un análisis de la gestión de los cuerpos y las emociones en algunos espacios de trabajo | Pedro Lisdero \& Diego Quattrini

se conforman bajo una regulación que permite la incorporación (aunque sea de manera tensionada) de modos de dominación establecidos. Estos procesos acontecen y se reproducen a partir de múltiples prácticas sociales, "imperativos morales", narraciones y visiones del mundo legítimas.

Así se instituyen diversas acciones (dentro y fuera de los tiempos/espacios de "trabajo") regidas por dispositivos que regulan emociones (miedo, resignación, esperanza, felicidad, etc.) y de mecanismos que las vuelven más "soportables" (que promueven la admisión de la vida social como "un-siempre-asi”" y el autocontrol para soportar el día a día laboral) (SCRIBANO, 2009). A su vez estos procesos de regulación están relacionados con las demandas generales de los empleos, es decir, con la normalización que producen las exigencias laborales en los sentidos y en la adecuación con el régimen de distancias y proximidades, que imparte el mundo del trabajo (QUATTRINI, 2012; LISDERO y QUATTRINI, 2013).

Por consiguiente, la gestión de las emociones en el trabajo obedece a las regulaciones y a las desregulaciones de las politicas corporales, configuradas a partir de una determinada "economía política de la moral" del capital ${ }^{5}$. Los dispositivos ideológicos son estructuras hechas prácticas que expresan los fundamentos de esta "moral hegemónica", que promueven y anulan sentidos orgánicos y necesidades. Así en el mundo laboral pueden identificarse "formas de soportabilidad", que aparecen como mediaciones, que permiten a los "excluidos" aceptarse como los dispensables del nuevo siglo; como también "maneras correctas de estar y apreciarse en el mundo", que mantienen la ilusión de la inclusión en el espacio competitivo del trabajo (LISDERO y VERGARA, 2010). Precisamente, estas transformaciones operadas desencadenan "nuevos" modos de asociatividad y gestión, que activan mecanismos que disminuyen la potencialidad disruptiva (LISDERO y QUATTRINI, 2013). La paciencia y la espera, el acostumbramiento en el trabajo (D`HERS, 2011), o la "percepción de obtener empleabilidad" (QUATTRINI, 2012), por ejemplo, emergen como maneras de sentir

\footnotetext{
${ }^{5}$ Con el término "economía política de la moral" hacemos alusión, siguiendo a Marx, al proceso de administración del conjunto de creencias, necesidades, deseos y acciones que promueven las formas capitalistas, articulando las conductas y las sociabilidades con las estructuras sociales (SCRIBANO, 2013).
} 
Trabajo y sensibilidades: Un análisis de la gestión de los cuerpos y las emociones en algunos espacios de trabajo | Pedro Lisdero \& Diego Quattrini

naturalizadas que afectan las disposiciones y las dimensiones subjetivas, sociales e incluso filogenéticas de los cuerpos, permitiendo la cesión de ciertos grados de resignación.

El cuerpo y las emociones se constituyen en un territorio en disputa, tanto por las propiedades y destrezas sociales como por ser un campo de acción insustancial, en el que se configuran las sensibilidades posibles. Así, identificar y analizar el juego que se produce en la materialidad del cuerpo entre sensaciones, percepciones y emociones para el trabajo es importante para entender las formas concretas en que se regulan las exigencias que afectan a los trabajadores. El cuerpo queda expuesto como objeto de sustracción y puesto en movimiento en el proceso productivo; configurando sensaciones y modos de actuación que garanticen respuestas a las interacciones y mandatos laborales.

\section{Experiencias paradigmáticas}

Las experiencias analizadas presentan distintos referentes empíricos, pero revelan los procesos de regulación del cuerpo y las emociones desarrolladas en el marco de la gestión del trabajo en el Sur/Global. El estudio de las particulares formas procedimentales, del control y de las experiencias asociadas a la práctica del trabajo posibilitan acercarse al posibilitan acercarse al examen de los procesos de construcción de sensibilidades sociales y a su mercantilización en estas latitudes.

\section{B.I. Los Call centers: el cuerpo y las emociones como claves analíticas en las "industrias sin chimeneas"}

Las transformaciones de la relación entre plusvalor y trabajo asalariado pueden analizarse de manera privilegiada en la expansión de los Calls Centers en Argentina. Estos últimos pueden ser entendidos como “(...) el soporte desarrollado por las empresas para satisfacer determinados aspectos de la relación con sus clientes en forma no presencial; son plataformas telefónicas que pueden ser gestionadas directamente por la empresa interesada en brindar atención a sus clientes, o por empresas proveedoras, en las que se ha tercerizado su gestión" (DEL BONO y BULLONI, 2008, p. 3). En efecto, la expansión de esta actividad a nivel mundial se vincula con la 
Trabajo y sensibilidades: Un análisis de la gestión de los cuerpos y las emociones en algunos espacios de trabajo | Pedro Lisdero \& Diego Quattrini

ampliación del outsourcing, por parte de las empresas transnacionales, a fin de reducir los costos de producción (OLIVERA, 2009; MONTARCÉ, p. 2011).

En Argentina, esta "industria" creció a partir de su impacto en la disminución de los costos laborales (se definió originalmente por Convenio Colectivo de Trabajo $\mathrm{N}^{\circ} 130 / 75$, como comercial lo que implicó una reducción adicional en comparación al personal telefónico). Para la primera década del siglo XXI, la actividad ya contaba con una concentración de empresas multinacionales proveedoras de servicios de exportación: menos de 10 firmas manejaban el mercado y, no más de 6 controlaban la venta de servicios hacia el exterior (DEL BONO, 2010). Se calcula que el sector ocupaba en ese tiempo 60 mil trabajadores, distribuidos en Buenos Aires, Rosario, Mendoza y Córdoba. Dichas ciudades, según representantes del sector, poseían como nicho de fuerza de trabajo una disponibilidad de jóvenes profesionales o estudiantes de niveles superiores.

La expansión de la actividad no estuvo desligada de manifestaciones conflictuales (al menos fragmentarias). En parte, esto se debe a que la tercerización diluyó las tradicionales relaciones políticas de los trabajadores, de modo que su representación se dividió progresivamente en varios sectores. Al mismo tiempo, las empresas, algunos sindicatos y el poder político desplegaron mecanismos jurídicos-legales-administrativos que judicializaron las potenciales protestas, negando las personerias gremiales a las nacientes organizaciones del sector. La invisibilización del conflicto puede rastrearse también en las dimensiones más sutiles de las relaciones cotidianas de trabajo, por la vía de las tecnologías organizacionales o el "management tóxico" (BRAGA, 2009). Junto a esto, la aplicación de los "estándares de calidad" en el servicio de atención, supone la intervención de mecanismos en la subjetividad de los trabajadores que performa sus miradas sobre sí mismos, afectando su identidad individual y colectiva.

La disposicionalidad requerida del sujeto, instruida en los "manuales de calidad", a partir del eufemismo de "sonrisa telefónica", expresa la injerencia tecnológica en el cuerpo sensible del trabajador. La palabra, las emociones, las capacidades más "intimas" de los sujetos, son minuciosamente intervenidas y la posibilidad de "decir-algo-distinto" ("sobre-sî" o, "por-sí- 
Trabajo y sensibilidades: Un análisis de la gestión de los cuerpos y las emociones en algunos espacios de trabajo | Pedro Lisdero \& Diego Quattrini

mismo"), en el diálogo que implica el servicio, se constituye en un desafío difícil de sortear, tal es el caso de la sonrisa, que está coercitivamente in-corporada.

La dimensión entre cuerpos/emociones y trabajo permite tramar algunos ejes analíticos. Podemos observar que en los Calls Centers hay un espacio representativo para indagar las formas de apropiación diferencial de la vitalidad de los cuerpos. El primer signo relevante, en este sentido, desmiente la "condición de inmaterialidad" como una forma vacía que describiría las experiencias de los escenarios emergentes del mundo del trabajo. Así como la digestión precisa de un estómago, la disponibilidad de energías para expropiar y ser puesta a disposición del aparato productivo del plusvalor (y reactivar así el mecanismo de expansión del capital) necesita de cuerpos. Pese a las diversas perspectivas teóricas que licúan la corporalidad, particularmente, en relación al avance e implicancia de las nuevas tecnologías en los procesos de trabajo, la energía productiva y la potencia de expropiación de la misma se basan en la existencia de un cuerpo biológico-orgánico y de la reconfiguración de la articulación cuerpo-tecnología dando lugar a "otras materialidades".

En apariencia, la expansión de los Calls Centers y las promesas asociadas a su "modelo laboral" promueven una idea de la inmaterialidad desconectada de las condiciones materiales de existencia con el trabajo, dado que éste suele caracterizarse como una tarea que no requiere esfuerzos para el sujeto (ni siquiera pensar, pues la charla está pautada, "guionada"). Emerge así la fantasía de un trabajador "emancipado" de condiciones materiales en su puesto: lo cotidiano para estos sujetos parece estar mediatizado exclusivamente por la información, es decir, por procesos digitales que se transforman en un continuo fluir de "energias inmateriales".

Dichas energías se anclan en la "charla-producto" de este trabajo, sometida a los procesos de "control de calidad" de la cadena de producción. Así, el trabajador constituido mediante los procesos teledigitales en "cuerpoinformación", moviliza datos en su actividad, produciendo una suerte de uniformidad de los discursos: "a nosotros nos pagan para leer los instructivos. Si vos no lees los instructivos, y das información errónea, te van a empezar a 
Trabajo y sensibilidades: Un análisis de la gestión de los cuerpos y las emociones en algunos espacios de trabajo | Pedro Lisdero \& Diego Quattrini

descontar dinero del sueldo" (Trabajador de una empresa de Calls Centers, Córdoba, 2010).

Es inherente a este proceso consagrar el borramiento del cuerpo del trabajador, en tanto "imposibilidad" de decir algo más allá de "lo pautado", o algo sobre sí mismo. En este sentido, la constitución de la identidad consagra esa apariencia de "tarea inmaterial". Sin embargo, la propia exigencia de productividad hace evidente el sustrato material que soporta la "charla". En este sentido, las demandas conflictuales del sector dejan más claro esto, rompiendo el idílico silencio que marca el borramiento de los cuerpos que trabajan, pues alertan sobre la redefinición de "lo material" en los actuales procesos de producción. Entonces, alli donde parecía que el sujeto se había liberado de tener que "ganar el pan con el sudor de su frente", bajo prescindencia del cuerpo en la producción de plusvalor, emerge un proceso de trabajo inseparable de las marcas que expropian la vitalidad de los cuerpos. La gran ausencia de éstos, en la aparente inmaterialidad de los "nuevos trabajos", es un indicador de la redefinición de la materialidad de su presencia.

Ligado a lo anterior, otra cuestión a analizar a partir de estas experiencias surge cuestionando la necesidad de pensar al propio cuerpo no sólo como bien en disputa, sino como territorio de una "guerra silenciosa" (LISDERO, 2012). En este sentido, la construcción social e histórica de la disponibilidad de un quantum energético, ligado a los cuerpos, se asocia a la expropiación de cierta capacidad operativa del ser social. Los rasgos extractivos y depredatorios de energías se constituyen en procesos que guardan relación con la coagulación y licuación de la acción social. Es decir, son mecanismos incorporados en los propios trabajadores, que contribuyen de manera adaptativa en sus escenarios laborales.

Para identificar estos mecanismos, se puede observar el despliegue de diversas emociones, cuyo saldo redunda en la naturalización/reproducción de las condiciones de expropiación. Por ejemplo, llama la atención en la actividad la recurrencia del miedo y su relación con los procesos de evitación del conflicto: "El tema que por ahí siempre hay miedo, de no reclamar lo que te corresponde... O por ahí somos medios quedados, no nos asesoramos, 
Trabajo y sensibilidades: Un análisis de la gestión de los cuerpos y las emociones en algunos espacios de trabajo | Pedro Lisdero \& Diego Quattrini

entonces nos conformamos en ese sentido, y seguimos, y seguimos, y así... (...). (Trabajadora de Calls Centers, Córdoba, 2009).

El miedo se define en articulación sinérgica con una serie de sensaciones complementarias, que performan la capacidad de acción; y se constituye en las vivencias naturalizadas de los trabajadores. Tiene su origen en la incorporación de las formas aceptadas de administrar las energías corporales; y opera a través de la organización de percepciones que configuran las sensaciones de amenaza, impotencia, extorsión e imposibilidad de desconectarse (de quebrar el destino expropiatorio). La amenaza se vivencia tanto en situación de protesta -donde se observaron manifestantes y marchas portando máscaras para no ser identificados-, como en las relaciones cotidianas de trabajo -donde los trabajadores son "controlados" a través de permanentes escuchas de las comunicaciones. Esta sensación opera como el complemento de una sensibilidad que paraliza. Las máscaras ocupan el lugar de lo que no se puede mostrar, de lo que se construye como invisible: otrosrostros de los trabajadores que permiten reconocerse como tales, otros movimientos que posibilitan quebrar el silencio cotidiano sobre el que se estructura el silencio aturdidor, otras formas de sentir que destituyen al "miedo" como "único destino posible". La conexión permanente, la amenaza constante operan como garantes de un miedo omnipresente. La vincha ${ }^{6}$ representa el silencio que emerge de una forma de "conexión", tramada por un estado de relaciones sociales que no soporta otra voz que la del cliente y la de la empresa, toda vez que la voz propia del sujeto trabajador tiene que ajustarse a los mandatos que regulan los manuales de calidad.

De esta manera, el miedo construido socialmente organiza las percepciones, estructura las "formas correctas de sentirse" que garantizan la "no-conflictividad" y las grandes tasas de ganancia para una industria que avanza sin voces que la interpelen.

A partir de la experiencia paradigmática del trabajo en los Calls Center, en clave de expropiación corporal, se observa el vínculo complejo entre las emociones (miedo, por ejemplo), su aspecto social y la soportabilidad de las

\footnotetext{
6 Vincha hace referencia al headset, o dispositivo con auriculares y micrófono, en tanto elemento de trabajo significativo del sector.
} 
Trabajo y sensibilidades: Un análisis de la gestión de los cuerpos y las emociones en algunos espacios de trabajo | Pedro Lisdero \& Diego Quattrini

condiciones de expropiación. Desde nuestra perspectiva, desentramar el funcionamiento de estos mecanismos contribuye a la comprensión de la relación entre la aludida metamorfosis del mundo del trabajo y los procesos que definen la expansión del capitalismo actual, pues, como observó Marx: “(...) lo que diferencia unas épocas de otras no es lo que se hace, sino cómo, con qué medios de trabajo se hace" (MARX, 2006, p. 218).

\section{B.2. Emprendedores de subsistencias: emociones para el trabajo en contextos de precariedad}

Al explorar la situación económica y social de los autodenominados emprendedores, se observa que la mayoría de los ellos asume su proyecto productivo como una "urgencia y una salida a su situación de desempleo". Muchos responden a un modelo de "autoempleo-cuentapropista", con niveles reducidos de ingresos que no pueden convertirse en salarios estables y menos en ganancias. Así lo explicaba en un diálogo en su casa, una emprendedora que trabaja en armados y difusión de páginas web: "cuando empecé estaba en un departamentito de dos ambientes con mis hijas, estábamos solitas porque todavia mi pareja estaba en Chile, no tenía escritorio, tenía la computadora en la cocina..." (Emprendedora diseñadora de páginas web, Villa María, 2016)

La inestabilidad y la precariedad laboral es común para quienes deciden emprender ante la necesidad de alcanzar ingresos para sobrevivir el día a día. Los emprendimientos de subsistencia son aquellos enmarcados preferentemente en la informalidad y se caracterizan por tener bajos niveles de tecnología, capital y productividad. Un artesano fabricante de lámparas de bambú, en una entrevista en su taller, comentaba sobre su definición de emprendedor en la que se incluía: “... un emprendedor es una persona que tiene una idea y quiere tirarla para adelante. Para mostrarla, venderla, comercializarla y crear como una empresa o una industria" (Emprendedor artesano, Villa María, 2015). En otras palabras, son ideas/proyectos que se ponen en práctica, enfrentando un sin número de problemas, a fin de suplir una necesidad económica personal/familiar.

Para convertir las ideas en una empresa, "mostrarla, venderla, comercializarla", no sólo se requieren conocimientos cognitivos sino la movilización de un "cuerpo sensible". El trabajo emocional, desarrollado bajo 
Trabajo y sensibilidades: Un análisis de la gestión de los cuerpos y las emociones en algunos espacios de trabajo | Pedro Lisdero \& Diego Quattrini

el régimen de sensibilidad, es necesario para promover nuevos movimientos corporales junto con sensaciones y estrategias de interacción adecuadas, que harán, al menos, más viable la sobrevivencia del emprendimiento. Ser constante, mantener una tolerancia a la frustración, el apego a la esperanza del crecimiento, son algunos estados emocionales que los emprendedores pueden y deben aprender a experimentar para manejarse en este contexto.

A continuación presentamos sintéticamente algunas características de las emociones, a partir de fragmentos de entrevistas en profundidad a emprendedores de subsistencia, de la ciudad de Villa María. La apertura de la charla se realizó luego de mostrarles a cada uno fotografias de sujetos en situación de trabajo que daban cuenta de tres emociones, representadas como actitudes, que se gestionan en el mundo laboral. Si bien las imágenes pueden adquirir diferentes significados de acuerdo con las experiencias de cada sujeto, la propuesta fue conectar en el diálogo las complejas relaciones entre trabajo y emociones, que se establecen en la informalidad de los emprendimientos.

Se le preguntó al artesano fabricante de lámparas de bambú, sobre la necesidad de ser "proactivo" y se le presentó una foto con un sujeto tomándose la cabeza, y alrededor de ella una serie de imágenes, tales como: un avión, una computadora, llaves, un reloj despertador, una señal de tránsito que dice "stop" y un edificio. El emprendedor contestó: "Sí, totalmente (me siento identificado)... porque tengo varias cosas en la cabeza y las tengo que manejar...lo que se muestra ahí lo peor que hay un mundo de ideas...Porque me sugiere ideas..."... "Y ser activo es estar en función de lo que uno hace. Dedicarle 24 horas... muchas horas en función de lo que uno quiere... Y depende... yo a veces me sueño haciendo lámparas... (risas)... Yo no trabajo solamente por cumplir con la tarea, tengo muchas ideas en la cabeza... y soy demasiado distraído... porque estoy todo el tiempo pensado en tantas cosas..." (Emprendedor artesano, Villa María, 2015).

Las fotos conducen al escenario del día a día del trabajo. En este caso pretende ser referencia a la relación entre el tiempo y las tareas que demanda el emprendimiento, como a las emociones que se construyen en torno a dicha relación. Aquí, el entrevistado marca la orientación de su acción, afirmando 
Trabajo y sensibilidades: Un análisis de la gestión de los cuerpos y las emociones en algunos espacios de trabajo | Pedro Lisdero \& Diego Quattrini

que sus energías corporales se mueven en función de un "mundo de ideas". Estas ideas poseen un repertorio de representaciones y percepciones que se construyen a partir de una experiencia motivacional, regida por un régimen de sensibilidad y un conjunto de mecanismos, que se van confluyendo en la definición subjetiva de ser emprendedor. Es una sensibilidad que se elabora a partir de estar activo todo el tiempo, conectado con los otros desde su negocio y pensando permanentemente en su producción. La proactividad se constituye en la "cabeza" del emprendedor, asumiendo una forma de ejecutar operaciones como una manera de ser y estar en el trabajo y un modo naturalizado de manejarse emocionalmente. Es un movimiento corporal, elaborado bajo un mandato productivo (trabajar las 24 horas), que produce sensaciones interpeladoras de los procedimientos temporales del trabajo. La percepción del sí mismo, de ser proactivo, queda articulada con una performance cotidiana y una gestión de estados sensitivos normativos.

La auto-motivación se activa a partir de sensaciones. No se trabaja solamente para cumplir con la tarea, sino además es necesario "ser seducido" moralmente, asumiendo la ilusión de la inclusión en el mundo de los negocios. En este sentido se observa la existencia de un plus emocional -en forma de esperanza- que sustenta las prácticas laborales habituales. Soñar, en este caso, origina un efecto de agilización de prácticas cognitivas que genera una re-significación de la responsabilidad y provoca formas de percibir y sentir adecuadas al régimen de flexibilización cotidiano del trabajo.

En otro sentido, otra emprendedora, diseñadora de páginas web, narra su impresión ante una imagen de un comensal, hombre de unos 40 años, quejándose por el plato de comida que le fue servido. Ella no se siente identificada con este retrato, aun así relata un discurso donde explica las implicancias emocionales que pueden producir los clientes en su trabajo: "Y esta foto, no me dice nada, que está comiendo, que tiene que ver con el cliente digamos... estando en un lugar así poniéndome en el rol del cliente no actuó de esa forma, no lo expreso de esa manera, en todo caso lo hablo tranquilamente con la persona que hay que hablarlo o me voy directamente, no confronto en ese sentido, si hay confrontación no se vuelve más..." (Emprendedora diseñadora de páginas web, Villa María, 2016) 
Trabajo y sensibilidades: Un análisis de la gestión de los cuerpos y las emociones en algunos espacios de trabajo | Pedro Lisdero \& Diego Quattrini

Aquí se observan las sensaciones provocadas por la imagen de un cliente descontento. Se identifica, en el relato, una forma en que se regulan las emociones frente a alguien disgustado: se prefiere no confrontar, es decir abordar la demanda "tranquilamente". La expresión de la tranquilidad aparece como una emoción vivenciada y decodificada a partir de experiencias particulares. El estar tranquilo es una capacidad que es alcanzada, a partir de una trama de sensibilidades construida y elaborada, según una significación regida por las reglas de estructuración del trabajo de servicio. Estas lógicas demandan apreciaciones cognitivas y prácticas que establecen y corren los limites de soportabilidad afectiva en el trabajo. El malestar y la frustración, que hace recordar la foto, está mediado por un tipo de valorización: en este caso, si la exigencia es decepcionante, el saldo mercantilizado de la emoción que produce la confrontación pierde su sentido para la emprendedora.

Se puede observar con la reacción de otro emprendedor a la misma foto otra trama de sensaciones. Un fabricante de aromatizadores, ante esta imagen, señala un conjunto de percepciones y emociones distintas: "A mí me hace recordar... que cuando yo arranqué el producto no era bueno... lo que pasa es que vos tenés que charlar con el cliente... es dificil... es el cliente que te da de comer... por eso es importante sobre todo la primera impresión... Y si yo caigo con la remera manchada y un montón de cosas, me va a decir que no venga más; porque no es lo mismo que yo vaya a un taller mecánico cambiado y estoy sucio al que vaya a un local de centro donde se vende zapatos y ropa..." (Emprendedor fabricante de aromatizadores, Villa Maria, 2016)

Emerge, en estas experiencias, la regulación de la impresión corporal, o más específicamente de la "primera impresión". La buena impresión del cliente es parte de las transacciones afectivas/estéticas que se producen en el trabajo. Se observa un sujeto que es permanentemente mirado desde su materialidad/inmaterialidad, demandando la estimación de un cuerpo actuante depurado. Asimismo hay un correlato entre las acciones, expresiones y modales con las emociones. En el cuerpo, se van condicionando ciertas visiones imperantes acerca de cómo ser y presentarse frente a los otros; lo que va regulando, a la par de los componentes físicos y simbólicos, las 
Trabajo y sensibilidades: Un análisis de la gestión de los cuerpos y las emociones en algunos espacios de trabajo | Pedro Lisdero \& Diego Quattrini

proximidades y distancias. La asunción personal "de no estar sucio" muestra otra característica de los procesos de estructuración del trabajo, aún en estos niveles de informalidad: la regulación de la presentación/imagen a través del sentido de la vista y de la interferencia de la estética, a partir de modos adecuados establecidos por el mercado (inclusive el no-formal): hay una necesidad de percibirse emocionalmente como una mercancía "atractiva" y "pulcra", para estar con los otros.

Si las experiencias, de los Call Center, alertan acerca de las marcas corporales que producen las renovadas cadenas de producción de valor y sobre los mecanismos y dispositivos de regulación emocional; por su parte el análisis de los cuerpos/emociones, de los emprendedores, deja visualizar la re-configuración de la dialéctica moderna entre los modos de percibir, de sentir y de expresarse, como sintoma de los procesos de estructuración asociados a las nuevas morfologías del trabajo.

\section{A modo de apertura}

Las complejidades que asumen nuestras sociedades locales en los comienzos del nuevo milenio exigen despojarse de miradas reduccionistas. En este sentido, hablar de la "metamorfosis del mundo del trabajo" constituye una propuesta teórica/ epistemológica necesaria tanto para abordar los procesos macro globales y su impacto en las configuraciones regionales, como en la regulación que se traza en los contornos de los cuerpos y sus sensibilidades de los habitantes del Sur/Global.

Este trabajo se orientó a generar un análisis de dos experiencias paradigmáticas, con dos referencias empíricas disímiles que de manera complementaria ponen de manifiesto algunos aspectos de la regulación de los cuerpos y emociones en el proceso normativo de la gestión del trabajo, como dimensión relevante de la metamorfosis aludida. Se observó cómo los renovados mecanismos disciplinares y procesos de naturalización van reconstruyendo nuevas prácticas identitarias, generadas en un mercado de trabajo regulado emocionalmente, como es en las experiencias de las actividades del cuentapropismo-emprendedor o del trabajo que se realiza en los Calls Centers. Esto supone una reconfiguración de una economía política 
Trabajo y sensibilidades: Un análisis de la gestión de los cuerpos y las emociones en algunos espacios de trabajo | Pedro Lisdero \& Diego Quattrini

de la moral, que proyecta modos de pensar, de sentir y de expresarse alrededor de un trabajo flexibilizado en sus cargas de tarea, dulcificado emocionalmente, extorsionado bajo la "presión laboral", y auto-responsabilizado, entre otras características.

Esta política de la moral puede ser analizada a partir de la relación entre las sensaciones vitales, que se van disputando en el espacio de trabajo, y las doctrinas empresariales legitimas de la administración científica, que procuran dar consejos sutiles y pautas para operar en la producción. El miedo, el malestar, la tranquilidad, la percepción estética del trabajador, aparecen como emocionalidades coactivas, gestionadas e envueltas en el proceso metabólico del capital, que se constituyen en un componente relevante para reformular el manejo discreto conductual y la estructuración de la cotidianidad laboral.

Los procesos de construcción de subjetividades, asociados a estos mecanismos, disponen del cuerpo y su sensibilidad como productor de capacidades. El cuerpo del trabajador debe presentarse provocando nuevos significados simbólicos para otros, en tanto debe aprender a "controlarse emotivamente", bajo nuevas experiencias sociales. Esto va produciendo una nueva moralidad, anclada en la presencia de mecanismos extorsivos, ya sea constituidos jerárquicamente a través de un neo-taylorismo (como en la experiencia de los Calls Centers) o bajo un régimen de chantaje emocional reproducido por los encargos y presiones del mercado informal y del consumidor-cliente (como sucede en la actividad del "emprender").

Las sensaciones reconstruidas en estas formas de trabajar tienen su relación con las tecnologías organizacionales empresariales. En el espacio de los Calls Centers se observa como la "doctrina" va interviniendo "minuciosamente" en las capacidades de los sujetos, más precisamente en su competencia de hablar y en el control de la palabra y la habilidad de expresarse. Precisamente la expresión material de la sonrisa queda impostada verbalmente, construida a partir de un momento coercitivo, mediado por los procesos digitales de información. A partir de allí se producen formas de administración de energías y de sensaciones corporales que deben ser 
Trabajo y sensibilidades: Un análisis de la gestión de los cuerpos y las emociones en algunos espacios de trabajo | Pedro Lisdero \& Diego Quattrini

aceptadas (a veces por el miedo), y que repercuten en la percepción identitaria del sí-mismo y en lo que puede hacer y no hacer en el trabajo.

Aquí se pretende anular el cuerpo del trabajador, limitándolo a un sujeto que produce un continuo fluir de bienes inmateriales/simbólicos. Sin embargo el proceso de gestión emocional es más complejo. La imposibilidad/posibilidad de decir algo propio o distinto va más allá de los objetivos y de las pautas normativas, que se imponen a partir de la injerencia de los procesos doctrinales. La marca personal/intima de sí mismo emerge de alguna forma. En la experiencia investigada, el uso de la máscara no sólo nos permite ver "lo que no se puede mostrar", sino que además esta experiencia vehiculiza otras emociones que posibilitan enfrentar al miedo y a la impotencia, generando otros movimientos e identidades laborales

La regulación del trabajo emocional en la experiencia de los emprendedores también se realiza a partir de la injerencia de ciertas creencias doctrinarias de la administración científica del trabajo, que ahora se reproducen en el marco de la informalidad. Los consejos emocionales se traman como una propuesta necesaria para la práctica de la sobrevivencia en el mercado. Los estados emocionales son necesarios para aprender a "manejarse" en un contexto de incertidumbre naturalizada y flexibilización laboral.

La proactividad se constituye como una capacidad emocional construida en la cabeza- que provoca estados sensitivos, que generan un movimiento corporal que extiende el tiempo productivo (aceptar el desafio de trabajar las 24 horas). La expresión de la tranquilidad (o la búsqueda de la misma) aparece como una emoción reconstruida y decodificada, a partir de experiencias acumuladas que permiten instaurar y correr los límites de la soportabilidad afectiva que requiere el trabajo (evitar como dé lugar a un cliente descontento). Al mismo tiempo, las transacciones afectivas/estéticas que se producen en la impresión, tensionan a un cuerpo sensible mirado por un otro que evalúa y estima su materialidad/inmaterialidad y normativiza el régimen de proximidades y distancias (para estar con el otro -el cliente- debo estar bien presentado). 
Trabajo y sensibilidades: Un análisis de la gestión de los cuerpos y las emociones en algunos espacios de trabajo | Pedro Lisdero \& Diego Quattrini

Detrás de estas intenciones gestuales/emocionales, al igual que en el espacio del teletrabajo, se constituye un cuerpo material configurado a partir de su exposición continua a una trama de sensibilidades, que van marcando su modo de ser y percibirse. Para elaborar el emprender es importante la propia capacidad de significación del sujeto, de su reconstrucción permanente de su visión cognitiva/valorativa del mundo, aunque siempre quede tensionada por las condiciones de estructuración del trabajo informal/precario.

En este sentido, el esfuerzo presentado en este artículo permitió además desentramar algunas pistas acerca de cómo se gestiona la diversidad a través de la producción, constituyendo un nodo clave para la acumulación actual. La radicalidad de estos procesos implica profundos cambios en la esfera de la producción de mercancías, y centralmente supone transformaciones en la propia constitución de la materialidad/inmaterialidad de los cuerpos. Precisamente, el trabajo, entendido como aquella práctica a la que las mayorias dedicaban gran parte de su vida diaria, comienza a adoptar otros modos y sentidos, des-constituyendo la rigidez de las dicotomías tales como la del trabajo/ocio, la empresa-fábrica/casa, el trabajo manual/trabajo inmaterial, lo público/privado, etc. Estas nuevas experiencias van repercutiendo, en la disputa de la disponibilidad de las energias que se configuran en las distintas actividades formales e informales.

Así, la relación cuerpo/capitalismo se constituye en un "indicador" del proceso de estructuración reciente, en tanto presenta la oportunidad de observar las dinámicas sociales que reconstruyen el proceso de revalorización capitalista. En este sentido, la economía política de la moral reinventa y reterritorializa mecanismos de expropiación, reconfigurando un mapa con nuevas coordenadas de poder. Aunque las transformaciones laborales se observen y se presenten en su alcance global, su impacto merece ser examinado a partir de las condiciones locales y regionales. Así, el saldo de la mercantilización de los cuerpos y emociones se constituye como una trama dialéctica entre las experiencias personales de los sujetos que trabajan, el protagonismo de la gestión doctrinaria productiva y, principalmente, las 
Trabajo y sensibilidades: Un análisis de la gestión de los cuerpos y las emociones en algunos espacios de trabajo | Pedro Lisdero \& Diego Quattrini

condiciones laborales precarias actualizadas y acopladas a un sistema globalizado.

Las sensibilidades sociales informan sobre las vivencias que se reconstituyen bajo las reconfiguraciones de los espacios. Las sociabilidades que surgen como saldo de las lógicas exploradas organizan las bases sociales para pensar los nuevos problemas de las ciencias sociales. El aporte específico de la sociología de los cuerpos/emociones a la comprensión de los fenómenos asociados a la transformación del trabajo es, desde la perspectiva que aquí se propone, un capítulo no menor en la construcción de una mirada crítica global sobre nuestras sociedades.

\section{Referencias:}

ACHA, Omar y HALPERIN, Paula (comps.) Cuerpos, géneros e identidades. Estudios de historia de género en Argentina. Bs As, Ediciones del Signo, 2000.

ANTUNES, Ricardo. (2006). La centralidad del trabajo hoy. Papeles de Población, Toluca, México, vol. 6, núm. 25, julio-septiembre, 2000

BERICAT ALASTUEY, Eduardo. La sociología de la emoción y la emoción en la sociología, Papers. Madrid, $\mathrm{N}^{\circ}$ 62, 2000.

BERICAT ALASTUEY, Eduardo. Max Weber o el enigma emocional del origen del capitalismo. REIS, 95, 2011.

BOURDIEU, Pierre. El sentido práctico. Madrid: Taurus, 1991.

BRAGA, Ruy. Vigança de Braverman: o infotaylorismo como contratempo. En ANTUNES Ricardo y BRAGA Ruy. Infoproletários. Degradaçao real do trabalho virtual, São Paulo: Boitempo, 2009.

CERVIO, Ana L. Las tramas del sentir. Ensayos desde una sociologia de los cuerpos y las emociones. Buenos Aires, ESE-Editora, 2012.

CITRO, Silvia y ASCHIERI, Patricia. Cuerpos en movimiento. Antropologia de y desde las danzas. Buenos Aires, Editorial Biblos, 2012.

DE LA GARZA TOLEDO, Enrique. Más allá de la fábrica: los desafíos teóricos del trabajo no clásico y la producción inmaterial, Nueva Sociedad. Buenos Aires, $\mathrm{N}^{\circ} 232,2011$.

DEL BONO, Andrea y BULLONI, Maria Noel. Experiencias laborales juveniles. Los agentes telefónicos de call centers offshore en Argentina. Trabajo y Sociedad, Santiago del Estero, $N^{\circ} 10,2008$ 
Trabajo y sensibilidades: Un análisis de la gestión de los cuerpos y las emociones en algunos espacios de trabajo | Pedro Lisdero \& Diego Quattrini

DEL BONO, Andrea. La geografia de los call centers: territorio, trabajo y empleo. En ROITMAN Susana, LISDERO, Pedro y MARENGO Leonardo. La llamada... El trabajo y los trabajadores de Calls Centers en Córdoba, Córdoba: Universitas, 2010.

D`HERS, Victoria. La materialidad de la sombra. Abyección y cuerpo en la definición de la basura. Revista Latinoamericana de Estudios sobre Cuerpos, Emociones y Sociedad, Buenos Aires, N5, 2011.

DURAND, Jean- Pierre. La cadena invisible: Flujo tenso y servidumbre voluntaria. México: Fondo de Cultura Económica. 2011.

ELÍAS, Norbert. E1 proceso de la civilización. Buenos Aires: Fondo de Cultura Económico, 1993.

FIGRI, Carlos. Eróticas de la disidencia en América Latina. Brasil, siglos XVII al XX. Buenos Aires, CLACSO-Ciccus, 2009.

FIGARI, Claudia. Hegemonía empresaria y nuevas lógicas de control social: La formación de mando. Trabajo y Sociedad, Santiago del Estero, N 17, 2011

GIDDENS, Antonhy. Modernidad e Identidad del yo. Barcelona, Península, 1991.

GOFFMAN, Erving. La presentación de la persona en la vida cotidiana. Buenos Aires: Amorrortu, 1989.

GREMILION, Helen. The cultural politics of body size. Annual Review of Anthropology, vol 35, 2005.

HABER, Stéphane. Cuerpos dominados, cuerpos en ruptura. Buenos Aires: Nueva Visión, 2007.

HARVEY, David. E1 nuevo imperialismo. Madrid: Akal. 2004.

HOCHSCHILD, Arlie Russell. The managed heart: commercialization of human feeling. California: University of California Press, 1983.

MARX, Karl. E1 capital. Tomo I/Vol 1, Libro primero. E1 proceso de producción del capital. Buenos Aires: Siglo XXI editores, 2006.

MATOSO, Elina (Comp.). E1 cuerpo In-cierto: arte/cultura/sociedad. Universidad de Buenos Aires y Editorial Letra Viva, 2006.

MONTARCÉ, Ines. Del otro lado del teléfono: identidad y Acción Colectiva. En DE LA GARZA TOLEDO, Enrique. Trabajo no clásico, organización y acción colectiva. Tomo II, México: Plaza y Valdés Editores, 2011.

NUN, José. 1969. La teoria de la masa marginal. Buenos Aires: Fondo de Cultura Económica, 2003

LE BRETON, David. La sociologia del cuerpo. Buenos Aires: Nueva Visión, 2002 . 
Trabajo y sensibilidades: Un análisis de la gestión de los cuerpos y las emociones en algunos espacios de trabajo | Pedro Lisdero \& Diego Quattrini

LISDERO, Pedro. La guerra silenciosa en el mundo de los Call Centers. Papeles del CEIC, Bilbao, vol. 2012/1, nº 80, 2012

LISDERO, Pedro y QUATTRINI Diego. Educación, Corporalidad y Nueva Morfología del Trabajo: Los Calls Centers ¿Escuelas de Trabajo? Intersticios: Revista Sociológica de Pensamiento Crítico, Madrid, Vol. 7 (1), 2013

LISDERO, Pedro y VERGARA Gabriela. Promesas y desencantos de los nuevos trabajos. Un análisis de los mecanismos de sujeción en los recuperadores de residuos. Pensamento Plural, Pelotas, Vol. 1, Nº6, 2010.

LISDERO, Pedro. Trabajo, cuerpo y conflicto social. La sonrisa telefónica y el trabajo emocional en los Call Centers. En ROBERTT Pedro, DÁVILA Ana Paula, LISDERO Pedro y BRAUNER FERREIRA Renata. O novo espitito do capitalismo no Sul: paralelismos e contrastes. Pelotas: Editora UFPel, 2017.

LISDERO, Pedro. Trabajo, cuerpo y conflicto social. La sonrisa telefónica y el trabajo emocional en los Call Centers. En ROBERTT Pedro, DÁVILA Ana Paula, LISDERO Pedro y BRAUNER FERREIRA Renata. O novo espitito do capitalismo no Sul: paralelismos e contrastes. Pelotas: Editora UFPel, 2017.

LUNA ZAMORA, Rogelio y SCRIBANO, Adrián. Contigo Aprendi. Estudios sociales sobre las emociones. Córdoba, Copiar, 2007.

OLIVEIRA, Sirlei Marcia de. Os trabalhadores das Centrais de Teleatividades no Brasil: da ilusão à exploração. En ANTUNES, Ricardo y BRAGA, Ruy. Infoproletários: degradação real do trabalho virtual. São Paulo: Boitempo, 2009.

PECHENY, Mario; FIGARI, Carlos, y JONES, Daniel (Comp.) Todo sexo es politico. Estudios sobre sexualidades en Argentina. Buenos Aires, El Zorzal, 2008.

QUATTRINI Diego, MARTÍN Rocío y RAIMONDO Heidi. (2017). El uso de la entrevista y la fotografia como técnica para el acercamiento a las emociones para el trabajo de los microemprendedores. En Gandía Claudia, Vergara Gabriela, Lisdero Pedro, Quattrini Diego y Cena Rebeca. Metodologia de la Investigación: Estrategias de Indagación I, Buenos Aires: ESEditora, 2017.

QUATTRINI, Diego. Prácticas, competencias y exigencias emocionales. Una mirada de los formadores de emprendimiento en Villa María. Revista Latinoamericana de Estudios sobre Cuerpos, Emociones y Sociedad, Buenos Aires, $\mathrm{N}^{\circ} 23,2017$

QUATTRINI Diego. Se busca trabajador... Un análisis del reclutamiento de trabajadores en la provincia de Mendoza. En COLLADO Patricia y TEIXEIRA DE SOUZA MARTINS Heloisa. Trabajo y Sindicalismo en Brasil y Argentina. San Pablo: Editorial HUCITEC, 2012.

QUIJANO, Aníbal. El trabajo al final del Siglo XX. Ecuador Debate. Clases Medias, Quito, $\mathrm{N}^{\circ} 74,2008$. 
Trabajo y sensibilidades: Un análisis de la gestión de los cuerpos y las emociones en algunos espacios de trabajo | Pedro Lisdero \& Diego Quattrini

SCRIBANO, Adrian. Policromia corporal. Cuerpos, Grafias y Sociedad. Córdoba, J. Sarmiento Editor, 2007a.

SCRIBANO, Adrian. Mapeando interiores. Cuerpo, conflicto y sensaciones. Córdoba, J. Sarmiento Editor, 2007b

SCRIBANO, Adrian. Sociologia de las emociones en Carlos Marx. North Carolina: Editorial A Contra Corriente, 2016

SCRIBANO, Adrian. La religión neo-colonial como la forma actual de la economía politica de la moral. De Prácticas y discursos, Resistencia, $\mathrm{N}^{\circ} 2$. Año 2, 2013.

SCRIBANO, Adrian. Teorias sociales del Sur: Una mirada postindependentista. Buenos Aires: ESEditora/E-Book Córdoba: Universitas, 2012 .

SCRIBANO, Adrian. A modo de epílogo ¿Por qué una mirada sociológica de los cuerpos y las emociones? En SCRIBANO Adrian y FIGARI Carlos. Cuerpo(s), Subjetividad(es) y Conflicto(s), Buenos Aires: CLACSO-Ciccus, 2009. 